\title{
1 Clinical data specification and coding for cross-analyses with omics data in
}

\section{2 autoimmune disease trials.}

3

4 Lorenzon Roberta ${ }^{1,2} *$, Drakos lannis ${ }^{1,2}$, Claire Ribet ${ }^{2}$, Sophie Harris ${ }^{2}$, Cordoba Maeva ${ }^{2}$, Tran

5 Olivia $^{3}$, Dasque Eric ${ }^{4}$, Cacoub Patrice ${ }^{5,1,2}$, Hartemann Agnes ${ }^{6,2}$, Bodaghi Bahram ${ }^{7,1}$, Saadoun 6 David $^{5,1,2}$, Berenbaum Francis ${ }^{8,2}$, Grateau Gilles ${ }^{9,2}$, Ronco Pierre ${ }^{10,2}$, Benveniste Olivier ${ }^{11,2}$,

7 Mariampillai Kuberaka ${ }^{11,2}$, Sellam Jeremie ${ }^{8,2}$, Seksik Philippe ${ }^{12,2}$, Rosenzwajg Michelle ${ }^{1,2}$, Six

8 Adrien $^{1,2}$, Bernard Claude ${ }^{2}$, Aheng Caroline ${ }^{2}$, Vicaut Eric ${ }^{3}$, Klatzmann David ${ }^{1,2, * *}$, Mariotti-

9 Ferrandiz Encarnita ${ }^{1,2^{* *}}$

$10 * * *$ these authors equally contributed to the work

111 Sorbonne Université, INSERM, UMR S 959, Immunology-Immunopathology-

12 Immunotherapy (I3); F-75005, Paris, France;

$12^{2}$ Biotherapy (CIC-BTi) and Inflammation-Immunopathology-Biotherapy Department (DHU

14 i2B), Hôpital Pitié-Salpêtrière, AP-HP, F-75651, Paris, France;

$15{ }^{3}$ Unité de recherche clinique, UMR 942, Univ Paris 07, Hôpitaux Saint Louis Lariboisière, 16 APHP, Paris, France;

$17 \quad{ }^{4} \mathrm{CIC}-1421$, Hôpital Pitié-Salpêtrière, AP-HP, Paris, France;

$18{ }^{5}$ UMR 974, UPMC, Department of Internal Medicine and Clinical Immunology, Hôpital Pitié19 Salpêtrière, AP-HP, Paris, France;

$20{ }^{6}$ Department of Diabetology, Hôpital Pitié-Salpêtrière, AP-HP, France; Faculty of Medicine, 21 Sorbonne Université; Institute of Cardiometabolism and Nutrition (ICAN), Paris, France;

$22{ }^{7}$ Département Hospitalo-Universitaire Vision and Handicaps 'ViewMaintain', Pitié23 Salpêtrière University Hospital, Paris , France

$24{ }^{8}$ Department of Rheumatology, Sorbonne Université, INSERM UMR_S938, Hôpital Saint25 Antoine, APHP, Paris, France;

26 '9 Sorbonne Université, INSERM, UMR_S 933, Department of Internal Medecine Hôpital 27 Tenon, APHP, Paris, France;

$28{ }^{10}$ Sorbonne Université; INSERM, UMR_S 1155, Paris, France; Hôpital Tenon, AP-HP, 29 Department of Nephrology and Dialysis, Paris, France; 
$30{ }^{11}$ UMR 974, UPMC, Department of Internal Medicine and Clinical Immunology, Hôpital Pitié-

31 Salpêtrière, AP-HP, Paris, France;

$32{ }^{12}$ Department of Gastroenterology, Hôpital Saint Antoine, AP-HP, and GRC-UPMC 03,

33 Sorbonne Université, Paris, France;

34

35 Keywords: multi-disease; harmonization; clinical trial; clinical coding

36 


\section{ABSTRACT}

38 Objectives: Autoimmune and inflammatory diseases (AIDS) form a continuum of 39 autoimmune and inflammatory diseases, yet AIDs' nosology is based on syndromic 40 classification. The TRANSIMMUNOM trial (NCT02466217) was designed to re-evaluate AIDs 41 nosology through clinic-biological and multi-omics investigations of patients with one of 19 42 selected AIDs. To allow cross-analyses of clinic-biological data together with omics data, we 43 needed to integrate clinical data in a harmonized database. Materials and Methods: We 44 assembled a clinical expert consortium (CEC) to select relevant clinic-biological features to 45 be collected for all patients and a cohort management team comprising biologists, clinicians 46 and computer scientists to design an electronic case report form (eCRF). The eCRF design 47 and implementation has been done on OpenClinica, an open-source CFR-part 11 compliant 48 electronic data capture system. Results: The CEC selected 865 clinical and biological 49 parameters. The CMT selected coded the items using CDISC standards into 5835 coded 50 values organized in 28 structured eCRFs. Examples of such coding are check boxes for 51 clinical investigation, numerical values with units, disease scores as a result of an automated 52 calculations, and coding of possible treatment formulas, doses and dosage regimens per 53 disease. Discussion: 21 CRFs were designed using OpenClinica v3.14 capturing the 5835 54 coded values per patients. Technical adjustment have been implemented to allow data 55 entry and extraction of this amount of data, rarely achieved in classical eCRFs designs. 56 Conclusions: A multidisciplinary endeavour offers complete and harmonized CRFs for AID 57 clinical investigations that are used in TRANSIMMUNOM and will benefit translational 58 research team. 


\section{BACKGROUND AND SIGNIFICANCE}

62 Autoimmune and auto-inflammatory diseases (AID) are the third cause of morbidity and 63 mortality in the world ${ }^{1}$. The development of more effective and better tolerated treatments 64 for these chronic and severely disabling diseases is an important public health issue. 65 Recently, genetic studies have highlighted altered biological processes that are common to 66 several $\mathrm{AIDs}^{2}$, and others studies have shown that an imbalance between effector $\mathrm{T}$ cells 67 and regulatory T cells resulting in the rupture of immune tolerance is associated with AIDs ${ }^{3-}$

$68{ }^{6}$. This collection of evidence is in line with the proposed reclassification of AIDs to form a 69 continuum of diseases ranging from pure autoimmune to pure inflammatory diseases with a number of diseases displaying variable degrees of both autoimmune and inflammatory disorders ${ }^{7}$. This is further sustained by immune markers common to several diseases, such as cytokines, which are currently targeted in therapeutics ${ }^{8,9}$. The complexity of these diseases, due to the various genetic and environmental factors as well as patient heterogeneity, prompted the scientific community to reconsider research practices with a view to a more integrative approach. In particular, AIDs are associated with multiple and variable immune-related disorders, including dysregulation of the innate immune response or of the adaptive immune response or of both. Systems biology approaches raise the hope that a more comprehensive understanding of cells and tissues in health and disease will open up new avenues for the treatment of patients ${ }^{10,11}$. These approaches will transform disease taxonomies from syndromic classification to molecular classification, and their combination, and will allow physicians to select optimal therapeutic regimens for individual patients ${ }^{12}$. Recent studies have successfully identified molecular signatures associated with specific autoimmune diseases ${ }^{4,13-16}$ as well as in physiological and pathological contexts ${ }^{17-}$ 19

85 Those results led us to setup an observational clinical trial, TRANSIMMUNOM, 86 (NCT02466217) the main goal of which was to revisit the nosology of AIDs through a 87 systems immunology approach. TRANSIMMUNOM participants include patients diagnosed 88 with one out of 19 selected AIDs or one out of 5 control diseases (Figure 1), and healthy 89 donors with no history of autoimmune disorders. The systems immunology approach used a 90 multi-scale deep immunophenotyping on peripheral blood (including transcriptome, TCR 91 repertoire, cytokine expression) and microbiome NGS studies. Importantly, classic routine 
92 biology assays as well as clinical investigations are fully part of the data collection strategy.

93 Our aim was to integrate all these data (biology, routine biology and clinical data) so as to

94 allow further cross-analysis of all patients and data to better characterize the immunome of

95 each patient regardless of the initial diagnosis. A similar strategy was initiated by the

96 National Institute of Allergy and Infectious Diseases (NIAID) under the Human Immunology

97 Project Consortium.

982 OBJECTIVES AND OUR CONTRIBUTION

99 Therefore, we needed to develop data integration approaches to efficiently record and 100 store collected data such that we could easily analyze them afterwards through 101 computational biology approaches. The first challenges of the project were to implement a 102 comprehensive case report form (CRF) covering all diseases in terms of clinical data and 103 biomarkers and to provide a user-friendly, vocabulary-controlled and not expensive 104 platform with standard vocabulary to record all data collected by the clinical assistant during 105 patient interviews. To meet these challenges, we assembled the multidisciplinary "Cohort 106 Management Team (CMT)" composed of clinicians from different specialties, nurses, biology 107 medical doctors, clinical trial methodologists, immunologists and computer scientists.

108 Here we present our electronic CRF (eCRF), designed using an open-source electronic data 109 capture (EDC) tool, capturing more than 5000 multiparametric coded values from 865 110 harmonized clinical and biological parameters per subject included in a multi-disease clinical 111 trial focusing on 24 diseases, 22 areas of clinical investigation and one vast set of routine 112 biology assays. Altogether, we believe that this effort could be of interest for small cohort 113 studies for which the commercially available eCRF services are not accessible.

\section{MATERIAL AND METHODS}

\subsection{Study population}

116 Patients with one of the following AIDS, of the AID continuum are recruited for 117 TRANSIMMUNOM trial (Figure 1): familial mediterranean fever (FMF), ulcerative colitis, 118 Crohn's disease, spondyloarthritis, uveitis, myositis (polymyositis, dermatomyositis, 119 inclusion-body myositis, necrotizing and anti-synthetase related myositis), ANCA-related 
120 vasculatis (Churg-Strauss' disease and granulomatosis with polyangiitis (ex Wegener), non-

121 ANCA-related vasculitis (such as Behçet's disease, cryoglobulinaemia and Takayasu),

122 rheumatoid arthritis (RA), type-1-diabetes and systemic lupus erythematosus (SLE). We also

123 included patients with diseases exhibiting symptoms similar to those of some AIDs but

124 linked to different gene mutations (control diseases), such as TRAPS and CAPS as a control

125 for FMF, or diseases with a similar autoimmune mechanism with overlapping

126 clinical/biological features, such as antiphospholipid syndrome (APLS) as a control of for SLE,

127 or degenerative diseases that do not have the same mechanism as AIDs such as

128 osteoarthritis for RA and muscular dystrophy for myositis. Finally, healthy volunteers are 129 included.

\subsection{Cohort Management Team}

131 Set up to interact with a Clinical Expert Consortium (CEC), a Cohort Management Team

132 (CMT) of biological experts, routine laboratory personnel, clinical trial methodologists and

133 clinical investigation centre harmonized the clinical and laboratory outcomes/results. The

134 CMT ensured that all required data are collected in an appropriate format for analyses and 135 that the questions are unambiguous. The computer scientist defined the data and metadata 136 structure required to minimize non-controlled data entry and to specify the expected 137 values. The overall design was supervised by an immunologist involved in the scientific part 138 of the clinical trial, who liaised between the clinicians and the computer scientist.

\section{$139 \quad 3.3$ Data collection for eCRF design and coding}

140 Each clinician received an Excel form to be filled in with the description of the item to be 141 recorded in a standardized manner: item ID, item value type (string, decimal); list of pre142 determined item values; item value unit (if applicable); item value range (if applicable). 143 Afterwards, all the data collected from the different specialties were grouped and 144 harmonized using CDISC standards.

\subsection{OpenClinica implementation}

146 Given the amount of data to be collected across 19 AIDs and 5 control diseases, OpenClinica 147 v3.14, an open-source CFR-part 11 compliant electronic data capture platform has been 148 selected for the design and capture of selected clinico-biological data. A test and production 
149 instances have been installed on dedicated and secured CentOS virtual machines with 16Go

150 RAM, 8 cores and 15 Go disk space each. OpenClinica's application server (Tomcat v9.0.6)

151 and database server (PostgreSQL v.9.5) parameters have been upgraded to fit multiple

152 simultaneous data entry and data extraction, in particular JAVA_OPTS for heap memory

153 have been upgraded to $8 \mathrm{Go}$ instead of $1 \mathrm{Go}$ Mo by default.

\section{$154 \quad 3.5$ Patient anonymization}

155 To have completely anonymized subjects who are also unique (no double entries for the 156 same subject in our database because of anonymity) we developed the Anonymized Subject 157 Unification (ASU) system as a completely autonomous system that can be used for any 158 clinical trial. Briefly, ASU takes advantage of a unique identifier of each subject (like Paris 159 Hospital patient number [NIP] or French healthcare registration number [INSEE]) to produce 160 a simple 4-letter code by using a one-way encryption technique.

1614 RESULTS

\subsection{OpenClinica as the compromise in designing a multi-disease} eCRF

164 The TRANSIMMUNOM observational trial targeted recruitment of 1,000 patients suffering 165 from one out of 24 diseases and healthy controls. During a single visit, patient medical 166 history and clinical investigations are performed together with the collection of samples 167 (blood, serum and feces) for further multi-omics analyses. The goal of the trial is to revisit 168 the nosology of AIDs by defining groups/clusters of patients based on clinical and molecular 169 signatures that cut across disease classification. To deal with the expected amount of 170 heterogeneous (such as disease severity scores, imaging data, biological measures) from 171 routine clinical investigations, and to allow the cross-evaluation of clinical and omics data, 172 we needed to develop an eCRF with a system that allows further omics data integration. We 173 selected OpenClinica (OC) as an electronic data capture (EDC) tool to support our eCRF 174 design. OC is an open-source CRF-part 11 compliant EDC able to design complex eCRFs for 175 large studies ${ }^{20,21}$. One of the major features of $O C$ is to rely on Clinical Data Acquisition 176 Standards Harmonization (CDASH) from the Clinical Data Interchange Standards Consortium 
177 (CDISC $)^{22}$, which allows the harmonization of clinical and biological data coding. Finally, OC

178 includes the mandatory validation of all recorded data to ensure data quality ${ }^{20}$. In addition,

179 the main strategy of TRANSIMMUNOM is to cross-analyze data from multiple AIDs, each of 180 which is usually characterized by particular clinical investigation records and biological data 181 measures. We anticipated the final cross-analysis, which would require the same 182 information for all the diseases. Finally, the eCRF had to follow regulatory guidelines and 183 Good Clinical Practices to ensure data entry, traceability and integrity throughout the 184 patient recruitment period. Although installation and implementation of $O C$ is not trivial, as 185 it requires computer science expertise and time, we decided to favour the landscape of 186 possibilities offered by OC to fulfil our study requirement.

\section{$187 \quad 4.2$ A multidisciplinary workflow ensuring the design of a robust} multi-disease eCRF

189 Expertise in different but converging fields was pooled in the CMTs, each of which 190 participated in a 3-step workflow to (1) define the protocol, (2) design and (3) validate the eCRF (Figure 2). The first step of protocol definition involved a Clinical Expert Consortium

192 (CEC) to define the list of items for all the patients with the aim of collecting exactly the same information regardless of the disease. All the clinical specialists together selected a sample of items per specialty so that the CRF was reasonably comprehensive and synthetic. Biology lab experts were also questioned to ensure the feasibility of sample drawing and of the required biology assays. Upon collection and validation of the actual items to be recorded, the specification of the database started with the design of an e-template where the computer scientist structured the information for each item by imposing the format of the data and metadata. Once the e-template was defined, we proceeded to the eCRF design: the CEC, in close collaboration with the computer scientist, designed the clinical coding of clinical investigation data following an unambiguous format for each item with maximized use of a predefined list of responses in order to avoid erroneous data entry.

203 Biology lab experts defined for each parameter measured the value type (string, integer, 204 decimal, Boolean), as well as the unit and range, when applicable. All the information was 205 summarized in a spreadsheet and converted by the computer scientist into a PostgreSQL 206 relational database following the OpenClinica structure. Finally, clinical research technicians 
207 evaluated the user-friendliness of the eCRF, the clinical research assistant evaluated the

208 item relationship constraints, and finally the CMT validated the eCRF with a patient "Zero"

209 simulation before release for production.

\section{$210 \quad 4.3$ An integrated multi-disease eCRF}

211 As AIDs belong to different medical specialities, the CEC comprised clinicians working in 212 rheumatology, internal medicine, gastroenterology, diabetology, ophthalmology, medical

213 biology, nephrology and genetics who ensured the feasibility of data collection in terms of

214 cost, patient morbidity and examination invasiveness. The list of information to be collected

215 for all the participants was organized in 4 categories. For each recorded item, we defined

216 the type of value such as free text field, free numerical field, automated calculation, check-

217 box, drop-down list and calendar/date field (Figure 3). The first group of CRFs was built

218 under the "Patient description" category and included classic clinical information required

219 to assess the biology and social environment of the patient. Altogether, we selected 70

220 items organized as 7 CRFs (Figure 3A \& Supplementary material). Each CRF collects 4 to 30

221 different items. The second set of CRFs focuses on "Common clinical monitoring" and was

222 organized as 5 CRFs collecting generic clinical data at the day of the visit and accounting in

223 all for 88 items (Figure 3B \& Supplementary material). The third category explore the

224 "Routine biology monitoring" (Figure 3C, Supplementary material \& Table 1) and covered a 225 wide spectrum of tests.

\begin{tabular}{|c|c|c|c|c|c|c|}
\hline Hematology & Biochemistry & Protein electrophoresis & $\begin{array}{c}\text { Urine } \\
\text { analysis }\end{array}$ & Immunochemistry & Genetic & Serology \\
\hline $\begin{array}{l}\text { Basophils } \\
\text { Eosinophils } \\
\text { ESR } \\
\text { Ferritin } \\
\text { Hematocrite } \\
\text { Hemoglobin } \\
\text { Iron } \\
\text { Leucocytes } \\
\text { Lymphocytes } \\
\text { MCH } \\
\text { MCHC } \\
\text { MCV } \\
\text { Monocytes } \\
\text { Neutrophils } \\
\text { Platelet } \\
\text { Red blood cells } \\
\text { Transferrin } \\
\text { Transferrin saturation }\end{array}$ & $\begin{array}{l}\text { 25-OH Vitamin D } \\
\text { Alkaline phosphatases } \\
\text { ALT } \\
\text { AST } \\
\text { Calcium } \\
\text { CH50 } \\
\text { Cholesterol } \\
\text { C-Peptide } \\
\text { CPK } \\
\text { Creatinine } \\
\text { Dyslipidemia } \\
\text { GGT } \\
\text { Glycemia } \\
\text { HDL } \\
\text { LDL } \\
\text { Phosphate } \\
\text { Triglyrecides } \\
\text { us-CRP }\end{array}$ & $\begin{array}{l}\text { Albuminemia } \\
\text { Alpha } 1 \text { globulin } \\
\text { Alpha } 2 \text { globulin } \\
\text { Beta globulin } \\
\text { Gamma globulin } \\
\text { Protein electrophoresis } \\
\text { peaks }\end{array}$ & $\begin{array}{l}\text { Creatinuria } \\
\text { Hematuria } \\
\text { Proteinuria }\end{array}$ & $\begin{array}{l}\text { ANA } \\
\text { ANCA } \\
\text { Anti-CCP } \\
\text { Anti-dsDNA } \\
\text { Anti-EJ } \\
\text { Anti-ENA } \\
\text { Anti-GAD } \\
\text { Anti-HM CR } \\
\text { Anti-IA2 } \\
\text { Anti-Jo1 } \\
\text { Anti-OJ } \\
\text { Anti-PL12 } \\
\text { Anti-PL7 } \\
\text { Anti-SRP } \\
\text { Anti-ZNT8 } \\
\text { ASCA } \\
\text { C3 } \\
\text { C4 } \\
\text { CH50 } \\
\text { Cryoglobulin } \\
\text { Anti-KU } \\
\text { Anti-MAD5 } \\
\text { Anti-MI2 }\end{array}$ & $\begin{array}{l}\text { B cell clonality } \\
\text { HLAB27 } \\
\text { HLAB51 } \\
\text { HLADR4 } \\
\text { HLADR8 }\end{array}$ & HIV \\
\hline
\end{tabular}




\begin{tabular}{l|l|l|l|l|l}
\hline & & & & $\begin{array}{l}\text { Anti-PM/Scl } \\
\text { Rheumatoid factor } \\
\text { Anti-TIF 1 gamma } \\
\text { Anti-RNP }\end{array}$ & \\
& & & & \\
\hline
\end{tabular}

\section{Table 1: List of routine biology assay in the TRANSIMMUNOM trial}

227 Table abbreviation legend: ALT - alanine aminotransferase, ANA - antinuclear antibodies, ANCA - anti228 neutrophil cytoplasmic antibodies, Anti-CCP - anti-cyclic citrullinated peptide antibodies, Anti-dsDNA - Anti229 double stranded DNA antibodies, Anti-EJ - anti-glycyl-transfer RNA synthetase antibodies, Anti-ENA - anti230 extractable nuclear antigens antibodies, Anti-GAD - anti-glutamic acid decarboxylase antibodies, Anti-HMGCR 231 anti-3-hydroxy-3-methylglutaryl-coenzyme A reductase antibodies, Anti-IA2 - anti-Islet antigen-2 antibodies, 232 Anti-Jo1 - anti-histidyl tRNA synthetase antibodies, Anti-Ku - anti- Ku antigen antibodies, Anti-MDA5 233 melanoma differentiation-associated gene 5 antibodies, Anti-MI2 - anti-Mi-2 antibodies, Anti-OJ - anti234 isoleucyl-tRNA synthetase antibodies, Anti-PL7 - anti-threonyl-tRNA synthetase antibodies, Anti-PL12 - anti235 alanyl-tRNA synthetase antibodies, Anti-PM/Scl - anti- nucleolar macromolecular complex PM/Scl, Anti-RNP 236 anti-nuclear ribonucleoprotein antibodies, Anti-SRP - anti-signal recognition particle antibodies, Anti-TIF1237 gamma - anti-transcriptional intermediary factor 1-gamma antibodies, Anti-ZnT8 -anti-zinc transporter 8 238 antibodies, ASCA - anti-Saccharomyces cerevisiae antibodies, AST - aspartate aminotransferase, C3 239 complement fraction 3, C4 - complement fraction 4, CH50 - total complement activity, CPK - Creatinine 240 phosphokinase, ESR - erythrocyte sedimentation rate, GGT - gamma-glutamyl transferase, HDL - high-density 241 lipoprotein, HIV - Human Immunodeficiency Virus, HLA-B27-B51-DR4-DR8 Human leukocyte antigen -B27-B51242 DR4-DR8, LDL - low-density lipoprotein, $\mathrm{MCH}$ - mean corpuscular haemoglobin, MCHC - mean corpuscular 243 hemoglobin concentration, MCV - mean corpuscular volume, us-CRP - ultrasensitive c-reactive protein.

245 These included biological assessment of organ function (liver, kidney, bone marrow) and of 246 inflammation state and safety, organized in 6 CRF and covering 91 parameters. Finally, the 247 last set of CRFs recorded "Disease-specific monitoring" data and was subdivided into 3 CRFs 248 (Figure 3D \& Supplementary material) capturing 616 items, including disease activity scores 249 as described in Table 2. This is thought to be as wide as possible in identifying clinical 250 parameters not usually collected in a particular disease including imaging and histology 251 features to allow the identification of disease profile, disease severity and features possibly

252 shared by diseases. Each clinician of the CEC identified a collection of features observed in 253 his/her specialty as classic or rare parameters. The CMT gathered all the parameters from 254 the different specialties and listed them in the clinical status and clinical evaluation CRFs. 255 Altogether, we selected 865 parameters to describe each patient regardless of the disease.

\begin{tabular}{lll}
\hline \multicolumn{1}{c}{ Disease } & \multicolumn{1}{c}{ Diagnostic criteria } & \multicolumn{1}{c}{ Activity score } \\
\hline $\begin{array}{l}\text { Familial Mediterranean } \\
\text { Fever/TRAPS/CAPS }\end{array}$ & Heller $^{23}$ & AIDAI $^{24}$ \\
Glcerative colitis & clinical and histological features & Mayo $^{25}$ \\
Crohn's disease & clinical and histological features & $\mathrm{HBI}^{26}$ \\
\end{tabular}




\begin{tabular}{|c|c|c|}
\hline Spondyloarthritis & $\begin{array}{l}\text { ASAS }^{27} \\
\text { Modified New-York criteria }^{28}\end{array}$ & BASDAI $^{29}$ \\
\hline Uveitis & non-infectious uveitis & NA \\
\hline Myositis/dystrophy & clinical and biological & NA \\
\hline Vasculitis & 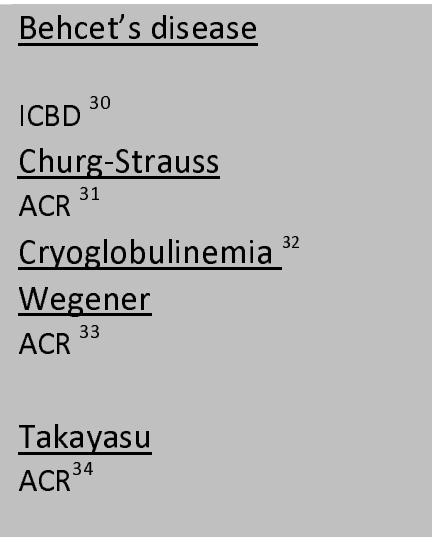 & $\begin{array}{l}\text { BVAS }^{35} \\
\mathrm{NIH}^{36}\end{array}$ \\
\hline Rheumatoid Arthritis & ACR; EULAR ${ }^{37}$ & DAS-28 ${ }^{39}$ \\
\hline Osteoarthritis & Kellgren-Lawrence 38 & \\
\hline Type-1-Diabetes & $\mathrm{ADA}^{40}$ & IDAA1C $^{41}$ \\
\hline $\begin{array}{l}\text { Systemic Lupus } \\
\text { Erythematosus / }\end{array}$ & $\mathrm{ACR}^{42,43}$ & SLEDAI ${ }^{45,46}$ \\
\hline $\begin{array}{l}\text { Anti-phospholipid } \\
\text { syndrome }\end{array}$ & Sapporo ${ }^{44}$ & \\
\hline
\end{tabular}

\section{$256 \quad$ Table 2: Disease specific diagnostic criteria and activity score}

\section{4.4 Clinical coding and CDASH harmonization}

258 Because of the heterogeneity of the selected parameters, clinical coding was designed as an

259 unambiguous format based on CDASH standards with maximized use of a predefined list of

260 responses, and was developed as a pragmatic, clinically-validated medical terminology with

261 an emphasis on ease-of-use data entry, retrieval and data analysis. We therefore defined

262 and validated for each parameter, wherever possible, the data-type (numerical, text, date,

263 predefined lists of options, value ranges) and units (when applicable) for all the parameters

264 identified in order to harmonize the information regardless of the collection time and

265 person and to avoid errors due to mistyping. Examples are Yes/No check boxes for clinical 
266 investigation, numerical values with a list of relevant units according to the parameter,

267 disease scores as a result of the automated sum of several scores, treatment description

268 including the coding of possible formulas, doses and dosage regimens (Table 3). We then

269 coded all the possible/expected values that each item could take and identified 1 to 8

270 possible variables per item coded as one of the value type. This work was especially critical

271 for the description of patient treatments. The list of all possible treatments regimen within

272 each specialty was fully generated with clinicians and is available in the database as a menu

273 list of 637 variables. Altogether, we built a database with 3815 uniquely coded variables.

274 However, since clinical status and evaluation of several diseases share identical CRFs, we

275 reached 5835 possible variables per patient. Altogether, we designed a collection of 21

276 CDASH harmonized CRFs recording 865 parameters with 5835 coded variables systemically

277 for all the patients and healthy donors included in the TRANSIMMUNOM trial.

\begin{tabular}{|c|c|c|c|}
\hline eCRF & $\begin{array}{l}\text { Coded } \\
\text { question }\end{array}$ & Coded answer & $\begin{array}{l}\text { Value } \\
\text { type }\end{array}$ \\
\hline Medical History & $\begin{array}{l}\text { Medical parameter } \\
>\text { AID family history }\end{array}$ & $\begin{array}{l}\text { Presence } \\
\text { YES or NO }\end{array}$ & $\nabla$ \\
\hline Hematology & $\begin{array}{l}\text { Clinical parameter } \\
>\text { Leucocyte count }\end{array}$ & $\begin{array}{l}\text { Numerical (min-max) | unit } \\
\text { Decimal }(4-10) \mid 10^{9} / \mathrm{L}\end{array}$ & 123 \\
\hline $\begin{array}{l}\text { Specific activity } \\
\text { score }\end{array}$ & $\begin{array}{l}\text { Disease } \\
>\text { Ulcerative colitis }\end{array}$ & $\begin{array}{l}\text { Disease score calculation } \\
\text { Mayo Score (Sum of } 3 \text { scores) }\end{array}$ & $\checkmark / x$ \\
\hline Treatment & $\begin{array}{l}\text { Medicine name } \\
>\text { Azathiopine }\end{array}$ & $\begin{array}{l}\text { Formula | Dosage | Posology } \\
\text { Tablet | } 25 / 50 \mathrm{mg} \mid 1 / 2 / 3 \mathrm{mg} / \mathrm{kg} / \text { day }\end{array}$ & \begin{tabular}{|l|l|l|}
$\nabla$ & 123 & $a b c$ \\
\end{tabular} \\
\hline
\end{tabular}

Table 3: Clinical item coding

279 For 5 exemplary eCRF, a coded question and expected coded answer are described with the type of values to

280 be entered following Figure 3 legend.

\section{DISCUSSION}

282 Clinical data management is of utmost importance for any clinical study. This includes

283 clinical information collection, validation and storage, usually completed through the use of

284 CRFs. While generally clinical research organizations (CROs) propose and use eCRFs, most

285 academic sponsored clinical studies still take advantage of the cost-benefit of paper versions

286 of CRFs, sometimes in combination with Excel based databases ${ }^{20}$. However, such tools,

287 although convenient, lack validation and data traceability. In addition, they do not usually 
288 use harmonized vocabulary and allow free text data entry. These drawbacks were

289 particularly counterproductive in our multi-disease clinical trial from several points of view.

290 First, the main goal of our trial is cross-analysis of multi-omics data obtained with clinical 291 and lab biology data from 1,000 patients selected for one out of 24 AIDs or control diseases.

292 Therefore, we needed an efficient and homogenized set of clinical and routine biology lab

293 for all the patients, which led to the selection of 865 parameters and to the coding of more

294 than 5000 values. This vast amount of data would have been unmanageable using classic

295 paper CRFs and spreadsheets. Second, the amount of information to be collected requires a

296 thorough validation with automated rules and limited free text data entry to avoid

297 mistyping and errors. Again, this cannot be handled using classic methods. Third, for cross-

298 analysis, we need to be able to extract clinical and routine biology data efficiently so that we

299 can filter for parameters as variables of interest (such as gender, BMI, disease activity,

300 autoantibody level). Again, considering the number of patients to be recruited in the

301 TRANSIMMUNOM trial, this would have been impossible. And finally, as regards the disease

302 heterogeneity, it would have been too expensive and complicated to ask an eCRF provider

303 to design such integrated CRFs. For all these reasons, we decided to take advantage of an

304 open-source EDC, Open-Clinica, for the implementation of our eCRFs. Although we

305 anticipated that the design and computer-based requirements would be time-consuming,

306 we found in this tool a number of advantages that allow (i) the integration of a very

307 significant amount of multi-parametric data, (ii) the possibility to design constraints rules

308 between entries to control data entry errors, associated with red flags in the case of errors

309 (for instance a man cannot be pregnant), (iii) the validation of the data entry by a third

310 person who double-checks (the latter advantages being CFR 21 - part 11 compliant) and (iv)

311 the addition of short instructions on the CRF page when needed to guide the data entry and

312 explain to the investigator how to fill in the eCRFs.

313 Altogether, this choice allowed the design of a controlled series of CRFs using harmonized

314 vocabulary to record data across 19 AID patients, 5 control disease patients and healthy

315 donors. This was made possible by the workflow we dedicated to the project, going from

316 the selection of parameters to be collected for all patients regardless of the disease to the

317 coding of all possible values per parameter in a harmonized manner based on CDASH

318 coding. 26 persons were involved in the process, including 14 clinicians, 1 computer

319 scientist, 7 scientists, 3 clinical research technician and assistant as well as 2 medical 
320 biologists for more than 100 hours of meetings and discussion over a year and a half. Clinical

321 data coding has the enormous advantages that it (i) pools reported terms in medically

322 meaningful groups, (ii) facilitates identification of common data sets for evaluation of

323 clinical information, (iii) supports consistent retrieval of specific cases or medical conditions

324 from a clinical database and (iv) smooths electronic data interchange of clinical safety

325 information.

326 Finally, our CRFs covers a wide spectrum of clinical and routine biology data of interest for

327 most AIDs, offering the community a pre-designed set of CRFs that can be used together or

328 individually. Although clinical safety was not added to our set of CRFs, because of the non-

329 interventional nature of the TRANSIMMUNOM trial, this could easily be done if needed. This

330 complex set of data has been harmonized and the database designed to store and query

331 efficiently the massive amount of data stored. Altogether, a truly multidisciplinary

332 endeavour led to the design and implementation a collection of 21 CRFs capturing more

333 than 5000 coded values that are now used in TRANSIMMUNOM and could benefit the

334 academic clinical community studying AIDs.

335 Acknowledgments: We are grateful to Frédéric Mariotti as an informatic subcontractor for

336 helping in the implementation and maintenance of the OpenClinica instance and to the

337 OpenClinica community, in particular Gerben Rienk Visser from Trial Data Solution for 338 providing help in OpenClinica parameter setup.

339 Funding: The work of RL, ID, CR, SH, CA, DK, EMF is funded by the LabEx Transimmunom

340 (ANR-11-IDEX-0004-02) as well as by Assistance Publique-Hôpitaux de Paris and Sorbonne

341 Université. TRiPoD funded the TCR-relevant part of the study.

342 Author contributions: RL, ID, CR, CA, AS, DK and EMF composed the Cohort Management 343 Team (CMT) for the design and implementation of the eCRF. RL, ED, PC, AH, BB, DS, FB, GG, $344 \mathrm{PR}, \mathrm{OB}, \mathrm{KM}, \mathrm{JS}, \mathrm{PS}, \mathrm{MR}, \mathrm{CB}$ formed the Clinical Expert Consortium (CEC) and defined the 345 selected clinical and biological data. RL and CR wrote the CRF in agreement with the CMT 346 recommendations. ID performed the computer science part of the work. CM, SH, TO worked 347 on the eCRF validation. EMF coordinated the design and implementation. EMF and RL wrote 348 the manuscript with input from all authors. EMF and DK conceived and supervised the 349 entire work.

350 Competing interests: Authors have no competing interests to declare. 


\section{$351 \quad 6$ FIGURES}

\begin{tabular}{|c|c|c|c|c|c|c|c|c|c|c|}
\hline & & IL-1 & IL-6 & IL-12 & IL-17 & IL-23 & TNF- $\alpha$ & IFN & Blys & B cell \\
\hline \multirow{7}{*}{ 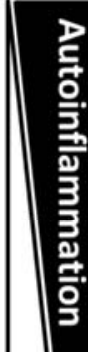 } & FMF & & & & & & & & & \\
\hline & TRAPS/CAPS & & & & & & & & & \\
\hline & IBD & & & & & & & & & \\
\hline & $O A$ & & & & & & & & & \\
\hline & $\mathrm{SpA}$ & & & & & & & & & \\
\hline & Uveitis & & & & & & & & & \\
\hline & Myositis & & & & & & & & & \\
\hline \multirow{6}{*}{ 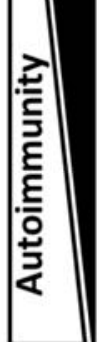 } & $M D$ & & & & & & & & & \\
\hline & Vasculitis & & & & & & & & & \\
\hline & RA & & & & & & & & & \\
\hline & T1D & & & & & & & & & \\
\hline & SLE & & & & & & & & & \\
\hline & APLS & & & & & & & & & \\
\hline & & $\begin{array}{c}\text { Anti- } \\
\text { IL-1 }\end{array}$ & $\begin{array}{c}\text { Anti- } \\
\text { IL-6 }\end{array}$ & $\begin{array}{l}\text { Anti- } \\
\text { IL-12 }\end{array}$ & $\begin{array}{l}\text { Anti- } \\
\text { IL-17 }\end{array}$ & $\begin{array}{l}\text { Anti- } \\
\text { IL-23 }\end{array}$ & $\begin{array}{l}\text { Anti- } \\
\text { TNF } \alpha\end{array}$ & IFN & $\begin{array}{l}\text { Anti- } \\
\text { Blys }\end{array}$ & $\begin{array}{l}\text { Anti- } \\
\text { CD20 }\end{array}$ \\
\hline
\end{tabular}

353 Figure 1: TRANSIMMUNOM selected AIDs and control diseases share immune markers and therapeutic strategies

355 This table shows the list of AIDs selected for the TRANSIMMUNOM trial, belonging to the AID continuum and their association with cytokines modulation (red) as well as immunotherapies targeting immune markers (grey). Abbreviation legend: Diseases: APLS anti-phospholipid syndrome, CAPS cryopyrin associated periodic syndromes, FMF familial mediterranean fever, IBD - inflammatory bowel disease, MD muscular dystrophy, OA osteoarthritis, RA rheumatoid arthritis, SLE systemic erythematosus lupus, SpA spondyloarthritis, T1D type 1 diabetes, TRAPS tumor necrosis factor receptor-associated periodic syndrome. Cytokines : IFN interferon, IL-1 interleukin-1, IL-6 interleukin-6, IL-12 interleukin-12, IL-17 interleukin-17, IL-23 interleukin-23, TNF- $\alpha$ tumor necrosis factor alpha. Immunotherapies: Anti-BLyS anti-BLyS monoclonal antibody, Anti-CD20 anti-CD20 monoclonal antibody Anti-IL-1 anti-interleukin-1 monoclonal antibody, Anti-IL-6 anti -interleukin-6 monoclonal antibody, Anti-IL-12 anti-interleukin-12 monoclonal antibody, Anti-IL-17 anti-interleukin-17 monoclonal antibody, Anti-IL-23 anti-interleukin-23 monoclonal antibody, Anti-TNF $\alpha$ tumor necrosis factor alpha-blockers. 
bioRxiv preprint doi: https://doi.org/10.1101/360719; this version posted July 5, 2018. The copyright holder for this preprint (which was not certified by peer review) is the author/funder, who has granted bioRxiv a license to display the preprint in perpetuity. It is made available under aCC-BY-NC-ND 4.0 International license.

367
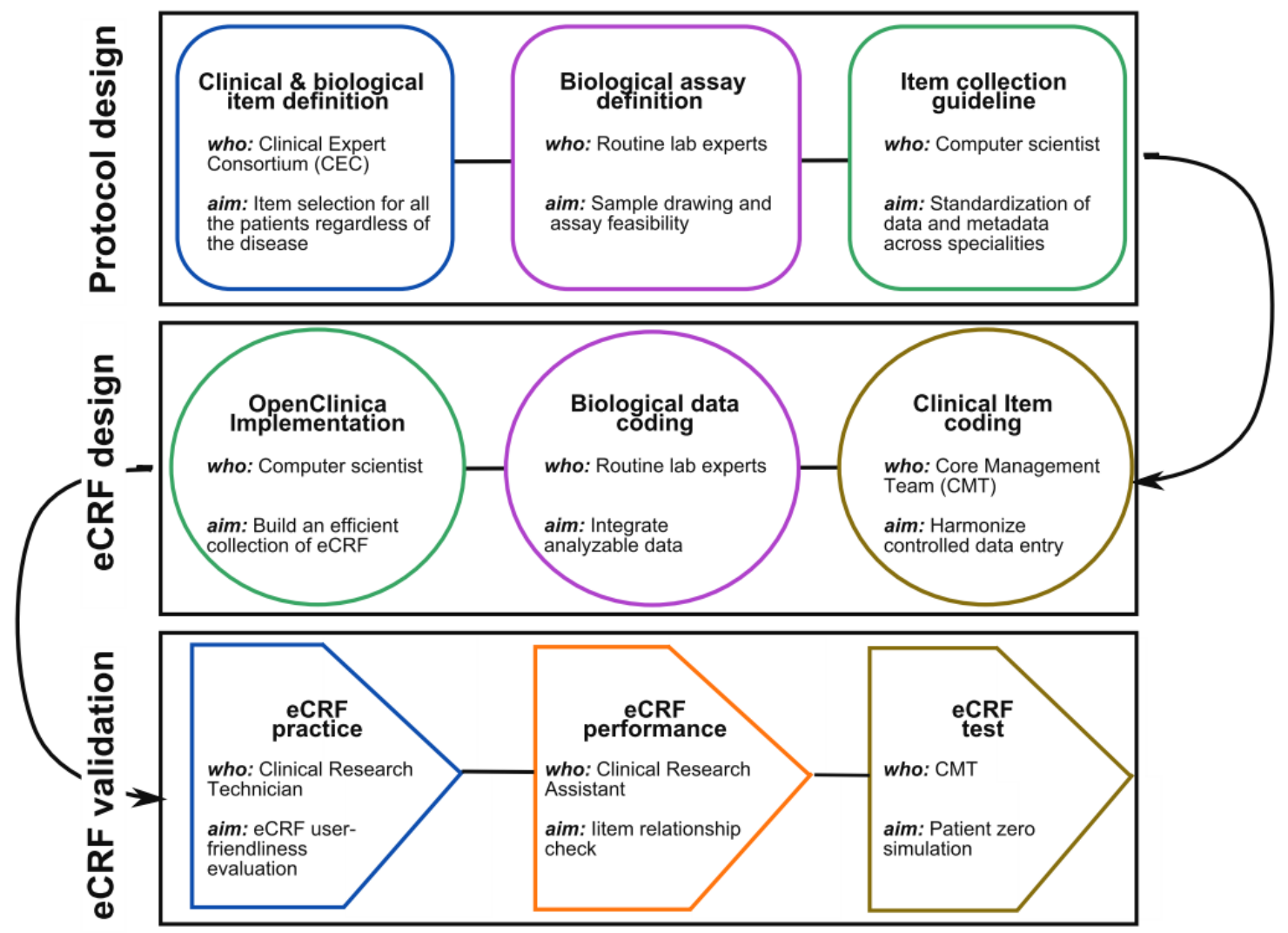

Figure 2: eCRf workflow

370

The figure represents the 3-steps workflow adopted for the eCRF design and implementation: (1) Protocol design, (2) eCRF design and (3) eCRF validation. In each box are listed the actions, its aim and the person in charge of it. Color code: Blue: clinical team, purple: biological team, green: computer scientist, and orange: the trial monitor team and brown the Core management team (see methods for description). 
A PATIENT DESCRIPTION

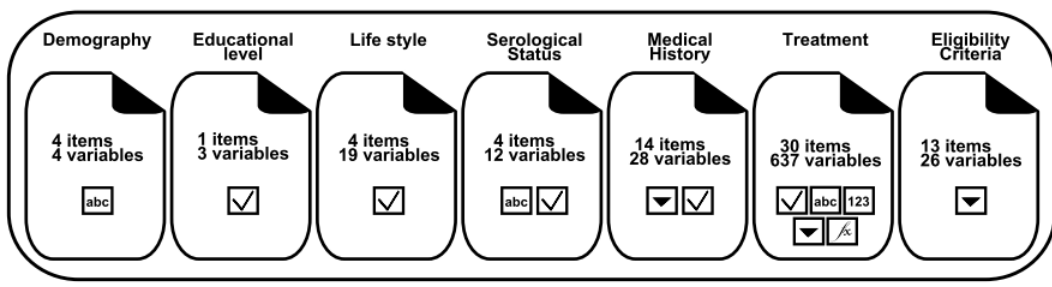

Legend

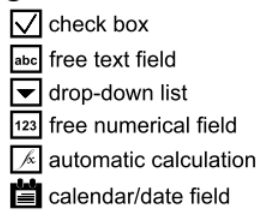

B COMMON CLINICAL MONITORING

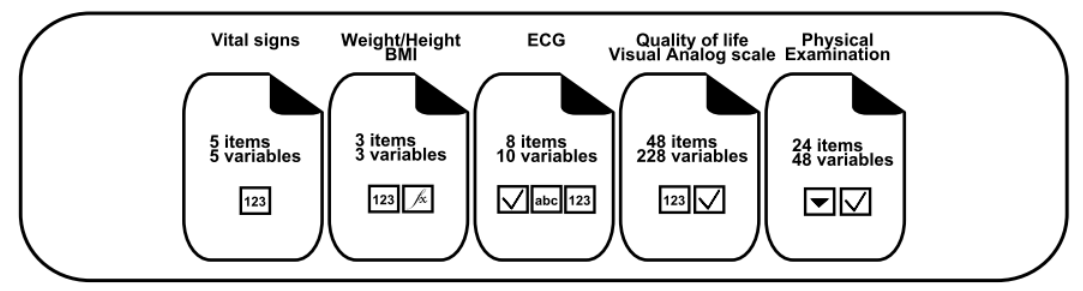

C ROUTINE BIOLOGY MONITORING

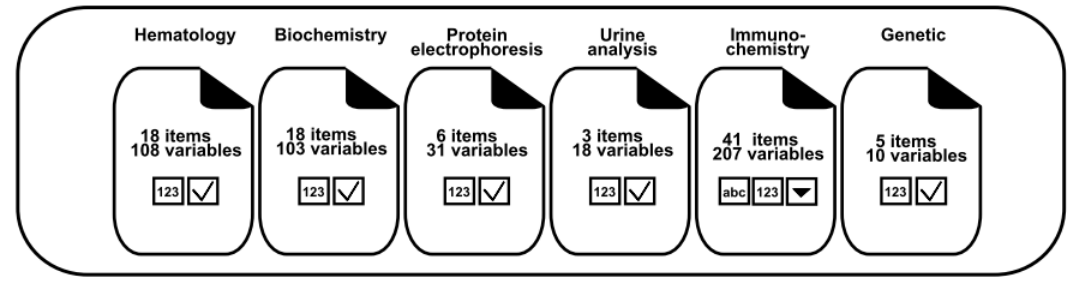

D DISEASE SPECIFIC MONITORING

375

376

377

378

379

380

381

382

383

384

385

386

387

388

389

390

391

392

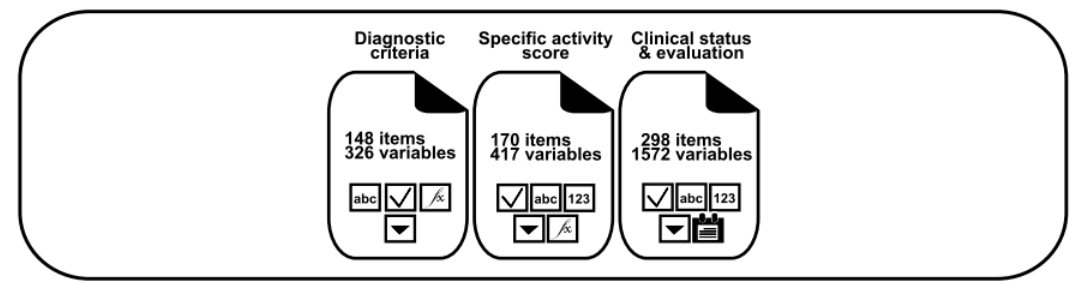

Figure 3: Schematic representation of the TRANSIMMUNOM integrated eCRF

Four categories of eCRFs were designed (A-D). Each category is composed of several eCRFs (form icon), each of which contained the indicated number of items for which 1 to 8 variables were coded. The type of values are indicated in the square boxes (see legend), so as to check-box, free text field, drop-down list, free numerical field, automatic calculation and calendar/date field. Altogether, 865 items were coded resulting in 5835 va riables organized in 21 eCRFs. See Supplementary material for details on eCRF

\section{REFERENCES}

1. Getts DR et al. Immunol. Rev. 255, 197-209 (2013).

2. Farh KK-H et al. Nature 518, 337-343 (2014).

3. Allenbach Y et al. Am J Pathol 174, 989-98. (2009).

4. Allenbach Y et al. PLoS One 9, e88788 (2014).

5. Buckner JH Nat Rev Immunol 10, 849-59 (2010).

6. Rosenzwajg M et al. Curr. Diab. Rep. 14, 553 (2014).

7. McGonagle D et al. PLoS Med. 3, (2006).

8. Magyari L et al. World J. Orthop. 5, 516-536 (2014).

9. Moran EM et al. Clin. Exp. Immunol. 178, 405-415 (2014).

10. Germain RN et al. Annu. Rev. Immunol. 29, 527-585 (2011). 
393 11. Pepperkok R et al. Genome Biol. 9, 314 (2008).

394 12. Bielekova B et al. Front. Neurol. 5, (2014).

395 13. Chaussabel D et al. Nat. Rev. Immunol. 14, 271-280 (2014).

396 14. Chaussabel D et al. Immunity 29, 150-164 (2008).

397 15. Saadoun D et al. N. Engl. J. Med. 365, 2067-2077 (2011).

398 16. Terrier B et al. Arthritis Rheum. 64, 2001-2011 (2012).

399 17. Godec J et al. Immunity 44, 194-206 (2016).

400 18. Nehar-Belaid D et al. J. Immunol. 196, 678-690 (2016).

401 19. Querec TD et al. Nat. Immunol. 10, 116-125 (2009).

402 20. Franklin JD et al. J. Biomed. Inform. 44, S103-S108 (2011).

403 21. Leroux H et al. Stud. Health Technol. Inform. 168, 89-95 (2011).

404 22. http://www.cdisc.org /CD

405 23. Livneh A et al. Arthritis Rheum. 40, 1879-1885 (1997).

406 24. Piram M et al. Ann. Rheum. Dis. 73, 2168-2173 (2014).

407 25. Lewis JD et al. Inflamm. Bowel Dis. 14, 1660-1666 (2008).

408 26. Harvey RF et al. Lancet Lond. Engl. 1, 514 (1980).

409 27. Rudwaleit M et al. Ann. Rheum. Dis. 68, 777-783 (2009).

410 28. van der Linden S et al. Arthritis Rheum. 27, 361-368 (1984).

411 29. Garrett S et al. J. Rheumatol. 21, 2286-2291 (1994).

412 30. International Team for the Revision of the International Criteria for Behçet's Disease (ITR-ICBD) J. Eur. Acad. Dermatol. Venereol. JEADV 28, 338-347 (2014).

415 32. Quartuccio L et al. Rheumatol. Oxf. Engl. 53, 2209-2213 (2014).

416 33. Leavitt RY et al. Arthritis Rheum. 33, 1101-1107 (1990).

417 34. Arend WP et al. Arthritis Rheum. 33, 1129-1134 (1990).

418 35. Stone JH et al. Arthritis Rheum. 44, 912-920 (2001).

419 36. Kerr GS et al. Ann. Intern. Med. 120, 919-929 (1994).

420 37. Aletaha D et al. Arthritis Rheum. 62, 2569-2581 (2010).

421 38. Kellgren JH et al. Ann. Rheum. Dis. 16, 494-502 (1957).

422 39. van der Heijde DM et al. Ann. Rheum. Dis. 49, 916-920 (1990).

423 40. American Diabetes Association Diabetes Care 35 Suppl 1, S11-63 (2012).

424 41. Mortensen HB et al. Diabetes Care 32, 1384-1390 (2009).

425 42. Petri M Rheum. Dis. Clin. North Am. 31, 245-254, vi (2005).

426 43. Hochberg MC Arthritis Rheum. 40, 1725 (1997).

427 44. Wilson WA et al. Lupus 10, 457-460 (2001).

428 45. Bombardier C et al. Arthritis Rheum. 35, 630-640 (1992).

429 46. Petri M et al. Lupus 8, 685-691 (1999). 